\title{
X-ray imaging beyond the limits
}

\author{
Henry N. Chapman
}

\begin{abstract}
The intense, brief pulses of X-rays from upcoming free-electron lasers will greatly extend X-ray microscopy to the femtosecond time domain and to interatomic length scales. From recent experiments and simulations one can envisage imaging macromolecules with X-rays without the need for crystallization.
\end{abstract}

n September 2009, the Linac Coherent
Light Source (LCLS) ${ }^{1}$ at the Stanford
Linear Accelerator Center (SLAC) in Linear Accelerator Center (SLAC) in
California will begin operations. This X-ray free-electron laser (XFEL) will then generate the brightest pulses of X-rays on the planet, leaping almost nine orders of magnitude beyond current synchrotron-based X-ray facilities. The LCLS, and other XFELs of its kind, will produce laser-like pulses of X-rays of about $100 \mathrm{fs}$ duration and $10^{12}$ photons per pulse, ushering in a new chapter in the field of ultrafast X-ray science. As with the introduction of the synchrotron, these new sources will have a great impact on many scientific disciplines, especially on structural biology, chemistry, atomic physics and materials science. Much of the new science in these fields will be guided by the improved capabilities in X-ray imaging that FEL sources will bring. Currently the dynamics of molecular and condensed matter systems are deduced from spectroscopy. Ultrafast $\mathrm{X}$-ray imaging will enable us to connect these studies with real space motions. Timeresolved X-ray crystallography, which is currently bringing new insights into the function of proteins, will be hugely extended with FEL pulses. The extreme intensity of the pulses will let us shrink crystal sizes all the way down to single molecules, giving three-dimensional movies of conformational dynamics and chemical reactions, and allowing the imaging of macromolecules that cannot be easily crystallized ${ }^{2}$.

The key to achieving ultrafast and extremely intense X-ray pulses in an XFEL is the process of self-amplification of spontaneous emission, which occurs when an electron pulse travels through a periodic magnetic structure called an undulator. The effect requires an extremely high peak charge of the electron pulse, obtained by compressing the pulses in a linear accelerator. The engineering feats involved in producing the electron pulses and feeding them through the 100-m long undulator, which must be aligned to the micrometre level, have been amply demonstrated. In fact, scientists at SLAC are confident enough in the process that time in 2009 for X-ray
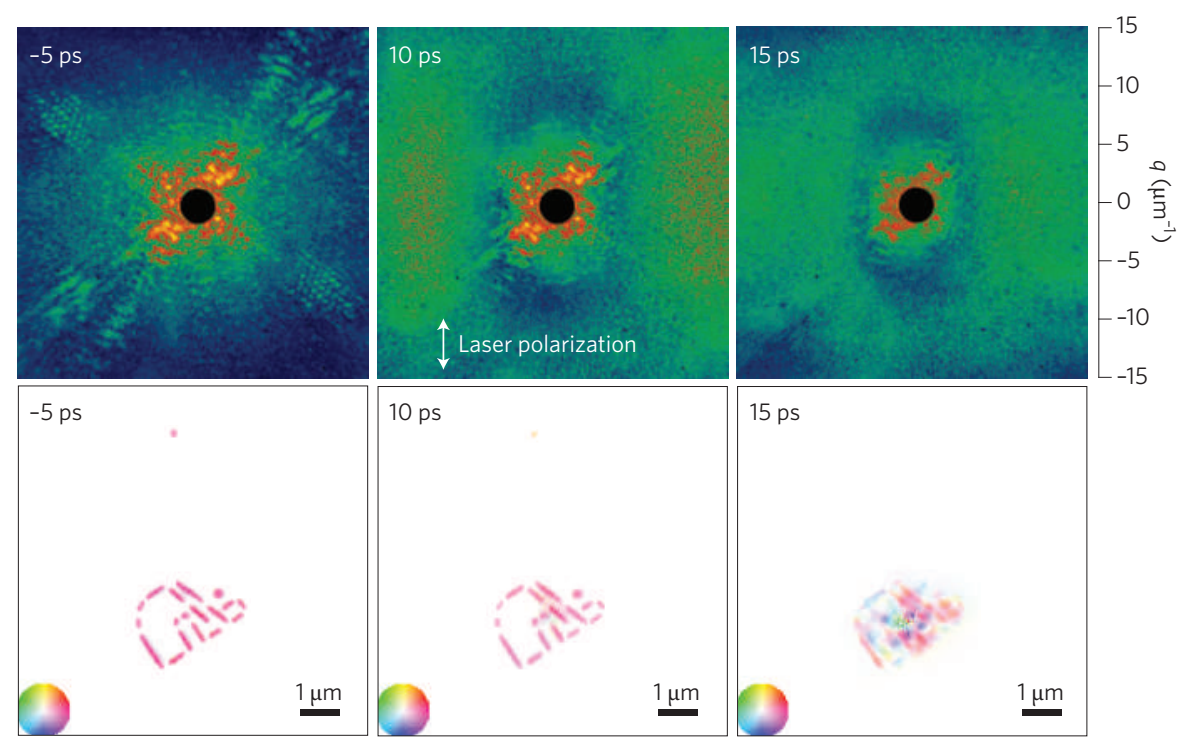

Figure 1 | Images of an exploding nanostructure. The top row shows ultrafast coherent diffraction patterns, and the bottom the reconstructed images ${ }^{11}$. The explosion of the structure was initiated by an optical laser pulse, which ablates the material, and recorded at the high resolution of the shortwavelength FEL pulses. Analysis of the patterns shows destruction propagating at the sound speed of $5,000 \mathrm{~m} \mathrm{~s}^{-1}$ until the resultant plasma expands and cools after a duration of $140 \mathrm{ps}$.

experiments has already been allotted. Other projects are gearing up: among them the Japanese XFEL ${ }^{3}$ at RIKEN in Japan, and the 3-km-long European XFEL ${ }^{4}$ in Hamburg are under construction. The FLASH facility at DESY in Hamburg, an extreme ultraviolet FEL which has been open for users since 2005 , is already demonstrating the new capabilities of these sources with pulses of duration of less than $30 \mathrm{fs}$, wavelengths in the range 6-40 $\mathrm{nm}$, and pulse energies higher than $100 \mu \mathrm{J}$ (ref. 5). Many improvements in FEL technology have been proposed even before the machines have been built, including methods of producing 1-fs pulses ${ }^{6}$, the timescale of atomic motions.

\section{Coherent X-ray imaging}

$\mathrm{X}$-ray FEL pulses are almost totally spatially coherent, which, along with the extreme intensities that can be achieved upon focusing, will enable X-ray microscopy to be extended beyond today's technological limits of X-ray objective lenses (see the review by Ade and Stoll ${ }^{7}$ in this Insight). Diffractive zone plate optics can be used to form images to about $20 \mathrm{~nm}$ resolution, although developments are required to extend their working distances to avoid their destruction by FEL pulses. Forms of lensless imaging, such as X-ray holography and coherent diffractive imaging, have been pursued as a way to overcome the limits of X-ray optics. Coherent diffractive imaging ${ }^{8}$ extends the ideas of phase retrieval in crystallography to reconstruct two- or three-dimensional images of non-crystalline objects from their diffraction patterns. In essence, if the coherent scattering pattern of a single object can be recorded, then the image can be constructed numerically, with the computer performing the task usually undertaken by a high-quality lens. The object must be illuminated with sufficient coherence that the scattered waves from extreme points in the object interfere to 
create a fringe pattern that encodes their separation. (Additionally, the pattern must be recorded on a grid fine enough to distinguish those fringes.) Sub-femtosecond resolution could be achieved owing to the inherent stability of the holographic geometries, to give exquisite measurements of lightmatter interactions $s^{9,10}$.

The potential of X-ray imaging at FEL sources is being explored at the FLASH facility. As a first proof-of-principle time-resolved imaging experiment at FLASH, the dynamics of laser ablation of a nanostructured silicon film was measured in transmission by coherent diffraction, demonstrating $50 \mathrm{~nm}$ spatial and $10 \mathrm{ps}$ temporal resolution ${ }^{11}$ (Fig. 1). In other experiments ${ }^{12}$ the optical 'pump' pulses that initiate dynamics have been synchronized to the FEL pulses with an accuracy better than $60 \mathrm{fs}$. These studies show that it is possible to use XFELs to study the structural dynamics of materials under extreme and non-equilibrium conditions, such as plasma formation, shock propagation in solids and crystals, or crack formation, and to investigate ultrafast dynamical processes in complex solids, such as magnetic switching and domain wall motion, phase transitions and protein function.

\section{Radiation damage}

There remains a question as to what changes are induced in the sample by the X-ray

a

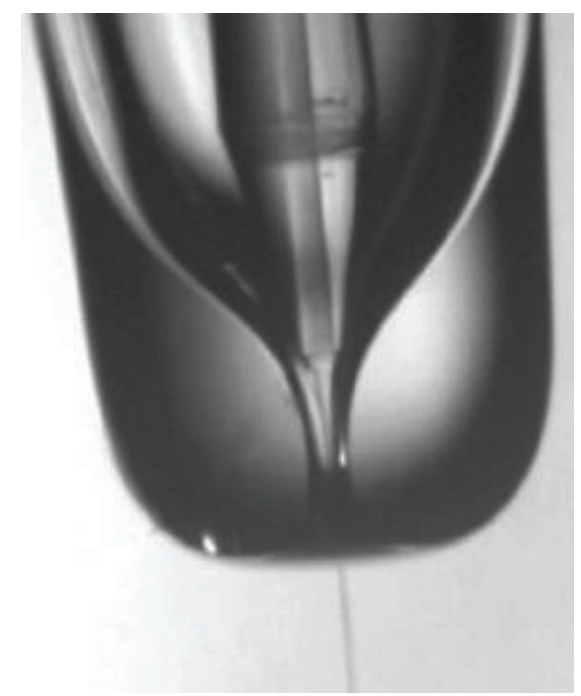

pulse itself, and what effect these changes may have on the measurement. X-ray pulse durations shorter than the Auger lifetime (2-10 fs, depending on the element) should beat electronic modification, although the effect on chemical shifts, near-edge resonant absorption and magnetic properties is not known. Stroboscopic measurements require the system to recover not only from the interaction of the stimulus but also from that of the X-ray probe pulse. Despite fears of XFEL pulses melting everything in their path, experimental conditions can certainly be arranged to minimize sample heating ${ }^{13}$. On the other hand, unprecedented X-ray intensities of $10^{19} \mathrm{~W} \mathrm{\textrm {cm } ^ { - 2 }}$ will be achieved by focusing the $0.15-\mathrm{nm}$ wavelength pulses to a $100-\mathrm{nm}$ spot, which will turn any material into a plasma, but ideally only after the pulse has traversed the sample. Complete destruction of the sample can be tolerated if the sample is renewed after each pulse (for example in imaging a shock wave in an impulsively strained crystal ${ }^{14}$, which could be made repeatable at the atomic scale), or if a single pulse on a single sample is sufficient to obtain an image. These are the premises of "diffraction before destruction" imaging ${ }^{15}$ to overcome radiation damage limits to imaging and to permit single-particle imaging of reproducible structures, such as macromolecules, without the need to crystallize them ${ }^{2}$.

b

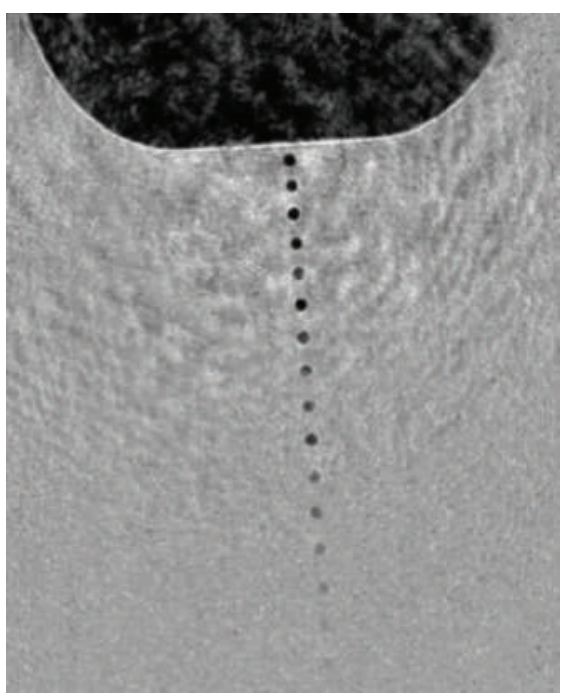

Figure 2 | Protein molecules, virus particles, nanocrystals and cells can be delivered in vacuum to a high-intensity X-ray FEL pulse, in droplets of water or other liquids. Such an environment could match the native conditions of the sample and provide a protecting mantle that delays its destruction. $\mathbf{a}$, One method is the aerojet developed by the Spence group at Arizona State University ${ }^{30}$. Here a coaxial gas flow decreases the diameter of the water jet before droplets are formed. $\mathbf{b}$, Drops can be synchronized by vibrating the nozzle with an ultrasonic piezoelectric transducer, as shown in this stroboscopic micrograph, with $25-\mu \mathrm{m}$ drops at a frequency of $169 \mathrm{kHz}$. Submicrometre-sized drops can be obtained. Figure reprinted with permission from ref. 30; (c) 2008 IOP.

\section{Unique objects}

The short X-ray pulses from FELs do indeed offer a way to overcome resolution limits caused by radiation-induced structural changes with current synchrotron-based biological X-ray microscopy. Biological materials such as cells and protein molecules are easily damaged by X-ray radiation ${ }^{16,17}$, setting a limit on how small a feature can be reliably imaged with 'long' exposures of many seconds. Smaller features obviously have a smaller scattering cross-section, requiring a higher dose. However, increasing dose causes ever more changes to the structure, owing to photoionization and the cascade of events that follows, leading in turn to a loss of image definition. It is thought that radiation damage will limit $\mathrm{X}$-ray imaging of protein in water to about $10 \mathrm{~nm}$ resolution ${ }^{17}$. Solem first suggested using pulses shorter than the timescale of destruction ${ }^{18,19}$, and calculated that under irradiation with an intense X-ray pulse the hydrodynamic explosion of the cell would be only a few nanometres over the duration of a 120 -fs pulse. More recent calculations ${ }^{20}$ have been made with a more complete model of the X-ray interaction. Obtaining the best resolution in a single-shot two-dimensional image is a balance between choosing a wavelength short enough to overcome diffraction limits yet making it long enough to increase the scattering cross-section of cellular components. Under these conditions the pulse intensity may be reduced so that the hydrodynamic motion during the pulse is also matched to this resolution. Including all these effects indicates that spatial resolution below $1 \mathrm{~nm}$ could be achieved on whole unsectioned cells irradiated by soft-X-ray pulses ${ }^{20}$.

But will it be possible to interpret an image in which one resolves 1-nm features throughout a cell that is 1,000 times thicker than that? These coherent images are much like digitally reconstructed holograms, and as such one can numerically focus through the object ${ }^{15}$. However, when the depth of focus is small, which is the case for imaging near the wavelength diffraction limit, the out-of-focus features interfere and lead to high-contrast 'speckles' that dominate the image ${ }^{21}$. There are ways to avoid these: use an incoherent method such as forming an image from the $\mathrm{X}$-ray fluorescence (selecting the atomic lines present before ionization, and hence damage, sets in), use a much shorter X-ray wavelength to obtain a projection image not dominated by diffraction, or create a stereo pair by recording images made at two orientations through the cell. Because the cell is destroyed in the process, the images must necessarily be collected at the same time by splitting the beam and recombining it at the sample ${ }^{22}$. 


\section{Reproducible objects}

Image resolution can be extended even further, perhaps to the interatomic length scale to resolve the secondary structure of proteins, by combining many diffraction patterns from single particles in a stream of identical structures, such as macromolecules, protein complexes or viruses ${ }^{2}$. These particles may be in a liquid droplet stream ${ }^{23}$ (Fig. 2), or brought into the gas phase by aerodynamically focusing an aerosol of the particles ${ }^{24}$. The destruction inflicted on a small isolated particle by the X-ray pulse is less than for extended objects, at least initially, because photoelectrons leave the particle without undergoing further collisions. However, within tens of femtoseconds the remaining positive charge leads to a Coulomb explosion. Because this excess charge is distributed near the surface of the particle, the explosion starts from the outside. Longer pulses can therefore be tolerated if the object of interest is embedded in a sacrificial layer, a so-called tamper consisting of light matter such as water or helium. Pulses of $50 \mathrm{fs}$ duration could give a damage-limited resolution of $0.2 \mathrm{~nm}$ (ref. 25).

Particles such as single macromolecules are very weak X-ray scatterers. Even when a pulse of $10^{12}$ photons is focused down to $100 \mathrm{~nm}$, fewer than 5,000 photons will be recorded in a diffraction pattern, and much less than a single photon per pixel at high scattering angles (see Fig. 3). If the objects were perfectly oriented with respect to each other, then the diffraction pattern could be simply accumulated until enough signal was acquired. A tomographic series could then be obtained by changing the particle orientation, perhaps by changing the polarization angle of an alignment laser beam ${ }^{26}$. Even the simplest of molecules could be imaged this way ${ }^{27}$, in order to observe reaction or dissociation dynamics in the time domain. In many cases the alignment will not be perfect, or might not be possible at all. This could be overcome if the orientation could be inferred from the diffraction pattern itself. Amazingly, such a feat could be possible even at the expected signal levels of single-particle diffraction of macromolecules. In fact, simulations show that patterns may be oriented with respect to each other when only $4 \%$ of the pixels of a detector receive any photon counts at all $^{28,29}$. This is achieved by using correlations in the noisy patterns to extract the few bits of information required to represent the orientation. Under these conditions, enough signal for a full three-dimensional reconstruction could be acquired with about $10^{7}$ diffraction patterns, which at the LCLS could be collected in 30 hours, and at the European XFEL in less than an hour, as long as the illumination conditions

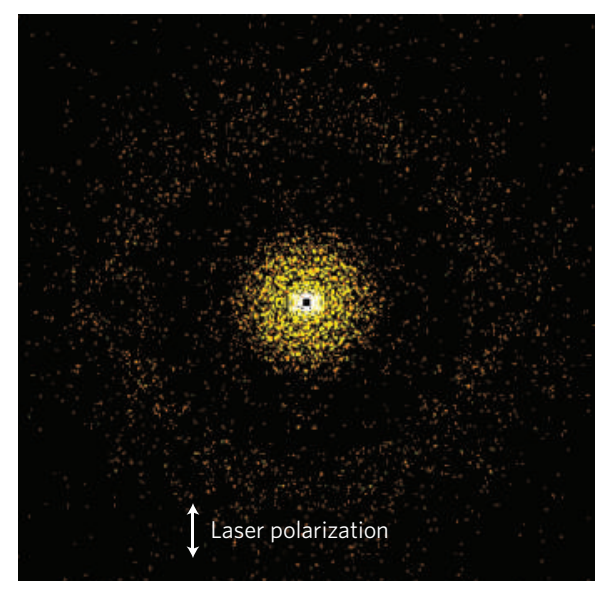

b

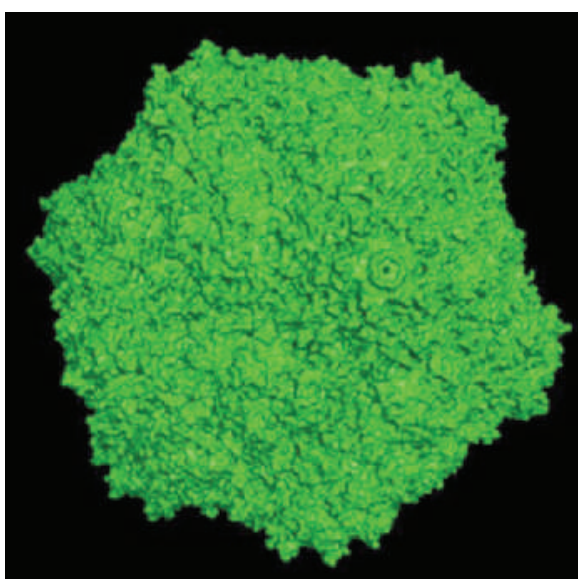

Figure 3 | Diffraction patterns from single objects differ from patterns of crystals consisting of repeats of those objects. The single-object pattern directly accesses the molecular transform without restriction to the crystal's Bragg peaks. a,b, The calculated pattern of the cowpea mosaic virus illuminated by a single LCLS pulse (a), consisting of $10^{12}$ photons focused to $0.2 \mu \mathrm{m}$ diameter. In this case the spotty nature of the pattern is due to the very low signal: most of the detector pixels would receive no counts (black) or a single $X$-ray photon (orange). Even so, new averaging algorithms should allow data to be combined from many patterns of different cowpea mosaic virus particles, recorded at random orientations, to reconstruct the three-dimensional image shown in $\mathbf{b}$.

remained constant from pulse to pulse.

These same algorithms could be applied to sort patterns not only into orientations but also into a range of conformers, or intermediate states in a reaction. They could also be used to assemble diffraction data from a stream of submicrometre-sized crystals, which often form in abundance but which are too small for synchrotron-based crystallography. Imaging single molecules will have far-reaching consequences, as many classes of proteins cannot currently be easily crystallized, including membrane proteins and large multi-protein assemblies. Obtaining images of these structures will greatly speed up therapeutic drug development and increase our understanding of the function and interactions of cellular components.

\section{A bright future}

Some of the first experiments planned for 2009 at LCLS will investigate just how far the capabilities of X-ray microscopy can be pushed into the time domain, and whether the high peak intensities will help us to obtain high-resolution X-ray images of macromolecules. X-ray FEL sources are expected to develop rapidly after that. The continued development of ultrafast $\mathrm{X}$-ray imaging, and its impact on our understanding of the basic interactions that determine functions in many areas from biology to superconductivity, will be driven by those developments. The future is indeed as bright as the $\mathrm{X}$-ray sources will be.
Henry N. Chapman is at the Centre for Free-Electron Laser Science, University of Hamburg, and DESY, Notkestrasse 85, 22607 Hamburg, Germany. e-mail: henry.chapman@desy.de

\section{References}

1. http://www-ssrl.slac.stanford.edu/lcls/

2. Neutze, R., Wouts, R., van der Spoel, D., Weckert, E. \& Hajdu, J. Nature 406, 753-757 (2000).

3. Shintake, T. et al. Nature Photon. 2, 555-559 (2008).

4. http://www.xfel.eu/

5. Ackermann, W. et al. Nature Photon. 1, 336-342 (2007).

6. Emma, P. et al. Phys. Rev. Lett. 92, 074801 (2004).

7. Ade, H. \& Stoll, H. Nature Mater. 8, 281-290 (2009).

8. Miao, J., Charalambous, P., Kirz, J. \& Sayre, D. Nature 400, 342-344 (1999).

9. Schlotter, W. F. et al. Opt. Lett. 32, 3110-3112 (2007).

10. Chapman, H. N. et al. Nature 448, 676-679 (2007).

11. Barty, A. et al. Nature Photon. 2, 415-419 (2008).

12. Cavalieri, A. L. et al. Phys. Rev. Lett. 94, 114801 (2005).

13. Grubel, G., Stephenson, G. B., Gutt, C., Sinn, H. \& Tschentscher, T. Nucl. Instrum. Methods Phys. Res. B 262, 357-367 (2007).

14. Robinson, I. \& Harder, R. Nature Mater. 8, 291-298 (2009).

15. Chapman, H. N. et al. Nature Phys. 2, 839-843 (2006).

16. Henderson, R. Q. Rev. Biophys. 28, 171 (1995).

17. Howells, M. R. et al. J. Electron Spectrosc. Rel. Phenom. doi:10.1016/j.elspec.2008.10.008 (2008).

18. Solem, J. C. \& Baldwin, G. C. Science 218, 229-235 (1982).

19. Solem, J. C. J. Opt. Soc. Am. B 3, 1551-1565 (1986).

20. Bergh, M., Huldt, G., Tî̀nneanu, N., Maia, F. \& Hajdu, J. Q. Rev. Biophys. 41, 181-204 (2008).

21. Cerbino, R. et al. Nature Phys. 4, 238-243 (2008).

22. Schmidt, K. E. et al. Phys. Rev. Lett. 101, 115507 (2008).

23. Weierstall, U. et al. Exp. Fluids 44, 675-689 (2008).

24. Bogan, M. J. et al. Nano Lett. 8, 310-316 (2008)

25. Hau-Riege, S. P., London, R. A., Chapman, H. N., Szoke, A. \& Timneanu, N. Phys. Rev. Lett. 98, 198302 (2007).

26. Spence, J. C. H. \& Doak, R. B. Phys. Rev. Lett. 92, 198102 (2004).

27. Ho, P. J. \& Santra, R. Phys. Rev. A 78, 053409 (2008).

28. Fung, R., Shneerson, V., Saldin, D. K. \& Ourmazd, A. Nature Phys. 5, 64-67 (2008)

29. Elser, V. Preprint at <http://arxiv.org/abs/0709.3858vl> (2007) 30. DePonte, D. P. et al. J. Phys. D 41, 195505 (2008). 